\title{
Experimental Optimization of a Free-to-Rotate Wing for Small UAS
}

\author{
Michael J. Logan, PE ${ }^{1}$; Richard DeLoach ${ }^{2}$; Tiwana Copeland ${ }^{3}$ \\ NASA Langley Research Center, Hampton, Va, 23681 \\ Steven $\mathrm{Vo}^{4}$ \\ California Polytechnic State University, San Luis Obispo, Ca, 93407
}

\begin{abstract}
This paper discusses an experimental investigation conducted to optimize a free-to-rotate wing for use on a small unmanned aircraft system (UAS). Although free-to-rotate wings have been used for decades on various small UAS and small manned aircraft, little is known about how to optimize these unusual wings for a specific application. The paper discusses some of the design rationale of the basic wing. In addition, three main parameters were selected for "optimization", wing camber, wing pivot location, and wing center of gravity (c.g.) location. A small apparatus was constructed to enable some simple experimental analysis of these parameters. A design-of-experiment series of tests were first conducted to discern which of the main optimization parameters were most likely to have the greatest impact on the outputs of interest, namely, some measure of "stability", some measure of the lift being generated at the neutral position, and how quickly the wing "recovers" from an upset. A second set of tests were conducted to develop a response-surface numerical representation of these outputs as functions of the three primary inputs. The response surface numerical representations are then used to develop an "optimum" within the trade space investigated. The results of the optimization are then tested experimentally to validate the predictions.
\end{abstract}

\section{Introduction}

$\mathrm{T}$ oday's users of small UAS typically are forced to choose between using limited endurance V/TOL craft or fixed-wing vehicles which impose significant limitations on operating areas. In both cases, users are limited in the types of weather environments they can operate within, usually meaning very benign wind conditions.

Typically, the platform of choice for V/TOL operations is some form of multi-copter. These craft have endurance typically limited to between 30 minutes and 1 hour. On the other hand, most fixed wing small UAS require significantly large takeoff and landing clear areas, even for hand launched versions. Further complicating the choice is that neither of these light, small UAS platforms handle high winds or gusty conditions well, thus limiting the operational utility of the whole genre.

In order to "bridge the gap" between the short endurance of current V/TOL platforms and their fixed-wing counterparts while simultaneously reducing the sensitivity of the platform to adverse wind conditions, a new type of wing was considered. This design is referred to as a "Compound Wing" because it has portions of the wing which are fixed, portions which articulate for V/TOL operation, and portions which are free-to-rotate about a span-wise axis. While the design of the fixed and articulating segments was relatively straightforward based on previous experience with small UAS design, the free-to-rotate segments presented some unique challenges to implementation.

\footnotetext{
${ }^{1}$ Head, NASA Small Unmanned Aerial Vehicle Laboratory, MS 238, Hampton VA, Senior Member.

${ }^{2}$ Senior Research Scientist, Aeronautics Systems Engineering Branch, MS 238, Hampton VA, Associate Fellow.

${ }^{3}$ Engineering co-op student, MS 238, Hampton, VA.

${ }^{4}$ Summer Intern, MS 238, Hampton, VA. 


\section{Initial Design Considerations}

During an extensive literature search, it was found that Free-to-rotate (FTR) wing concepts have been in existence since the mid-forties. An early patent (Ref. 1), by D. R. Zuck, describes a light general aviation aircraft with a wing that pivots along a spanwise axis. There was a brief look at a similar concept in the late-sixties (Ref. 2), although the wind tunnel tests conducted were limited in scope and did not provide a sufficiently broad understanding of the design parameters of interest. There was a series of analytical studies looking at small-to-large general aviation aircraft with free-to-rotate wings (Ref. 3,4). These studies tended to focus on development of an analytical framework and were very specific to the mission and vehicle class being evaluated. NASA conducted some flight tests of a free-to-rotate radio-controlled model where the free-to-rotate wing had an attached canard "trimmer" in the mid-to-late 70s (Ref. 5,6,7). The trimmer was found to induce a number of unpleasant attributes making normal R/C flight exceptionally difficult. There was a small company, Freewing, which built and sold some mid-sized UAS which utilized a free-to-rotate wing and a "tilting body" to provide a S/TOL takeoff and landing. Two universities were recipients of these platforms and have conducted some flight research with them.

Even though there is a significant past history of specific implementations of free-to-rotate wings, there is not a good data set that examines the various necessary design parameters and how best to optimize those for a given desired outcome. For example, where should the pivot location be along the chord? Where should the wing c.g. be made to lie? What is the impact of changing the airfoil section characteristics on the stability, damping, lift and drag of the wing? The answers to these basic questions appears to have been "we used the same design those other folks did before us" in the literature. This was an unexpected result and led to the need to conduct some form of limited experimental approach to help determine what variations in those parameters cause in the vehicle's (or at least the wing's) basic performance.

\section{Experimental Setup}

An experimental setup was created which would allow the rapid testing of a large number of configurations of the three primary variables under consideration: pivot location, center of gravity (c.g.) location, and wing camber. A simple test apparatus was constructed consisting of a large diameter drum fan, a set of tubular "flow straighteners", and a box-like "flow accelerator" to provide a roughly $15 \mathrm{mph}$ flow test section. The experiments used a set of $2 \mathrm{ft}$. span, 10 in. chord airfoils, the same size as would be used on the UAS being designed so that the results would be directly applicable. (See Fig. 1) As part of the experiment, a servo was added to the wing to drive a trailing edge flap that could be deflected to determine the effect of same. Scales were placed under the columns supporting the pivot shaft to measure lift for each of the configurations tested. The wings were free to rotate around this pivot shaft. Measurement of the response to "gusts" was simulated by using a fixed deflection of the wing trailing edge (2") followed by release and observation of the result.

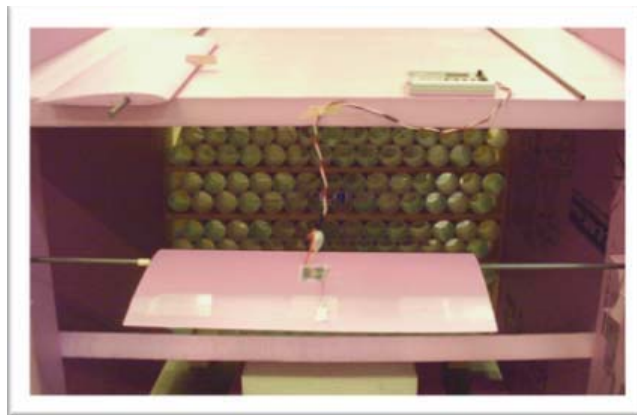

Figure 1. Test apparatus. 


\section{Experiments Performed}

In order to generate the most broadly applicable experimental test data base, three main independent variables were chosen to evaluate - airfoil camber (in percent), pivot location (as a percent of wing chord), and c.g. location of the rotating segment (as a percent of wing chord). Each of these primary variables were assigned a range of possible values based on most likely limits. A Design-of-Experiment process was used to develop which specific combinations of wing pivot location (from $15 \%$ to $30 \%$ chord), wing c.g. location (from $15 \%$ to $30 \%$ of chord), and wing camber (from 1 to 5\%) to test. A total of 22 runs were made as shown in Table 1 . Note that some were repeat points to check for variability in the measurements. To test the response to a gust, the trailing edge was deflected by 2 " and then released. A "stability" parameter was then measured that indicated the magnitude of the oscillations, a "recovery" parameter was measured that indicated the number of oscillations before returning to the original "neutral" point. In addition, the basic lift of the wing was measured with the trailing edge flap at the zero deflection setting. The "neutral” angle of attack was also measured.

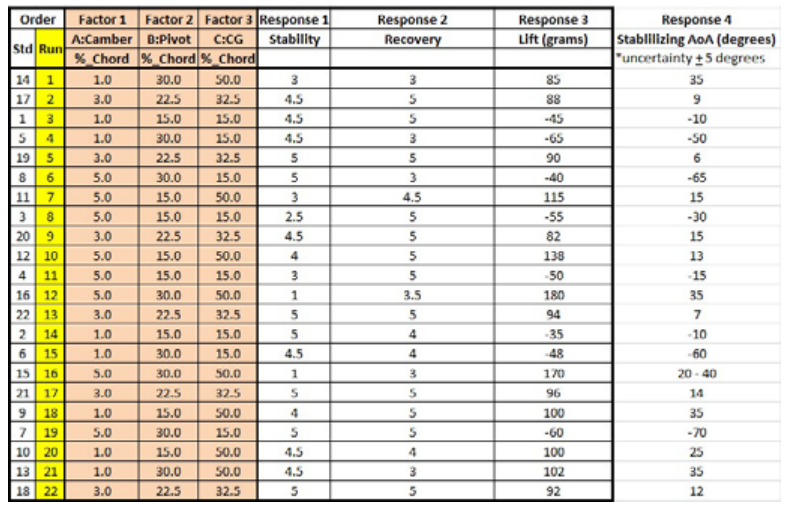

Table 1. Experimental results from initial experiment set.

Initial analysis of the experimental data indicated that there was a high correlation between increasing c.g. location and increasing lift. Further, it appeared that the lift generation wass relatively insensitive to pivot location. The "recovery" parameter appears to be most sensitive to pivot location whereas the "stability" parameter appears to have complex interactions between all three parameters. In addition, post experiment analysis indicated there was little sensitivity to wing camber in any of the key "output” measurements.

A second set of experimental measurements was conducted with only two primary indepenedent variables, pivot location and panel c.g. In some cases, the c.g. location selected by the experiment design utility were well within the measurement capability of the method used to determine panel c.g. In those cases, one c.g. location was used for those data points (42.2 and 42.5 both used 42.5 , for example). The experimental results from these measurements is shown in Table 2. 


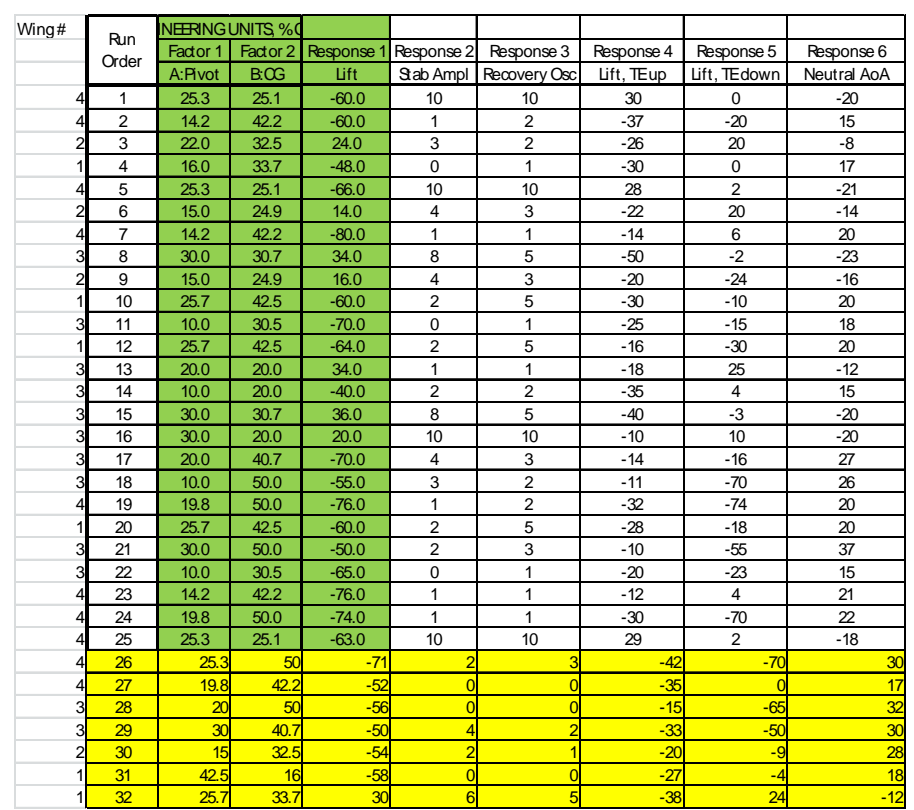

Table 2. Experimental results from second experiment set.

Analysis of the data suggests that there appears to be areas of "optimality" in terms of the pivot location and the c.g. location. There also appears to be competing issues in that although the lift at neutral angle-of-attack tends to get higher when the c.g. is farther aft, the apparent roll authority (the lift with the trailing edge up vs. trailing edge down) appears to decrease.

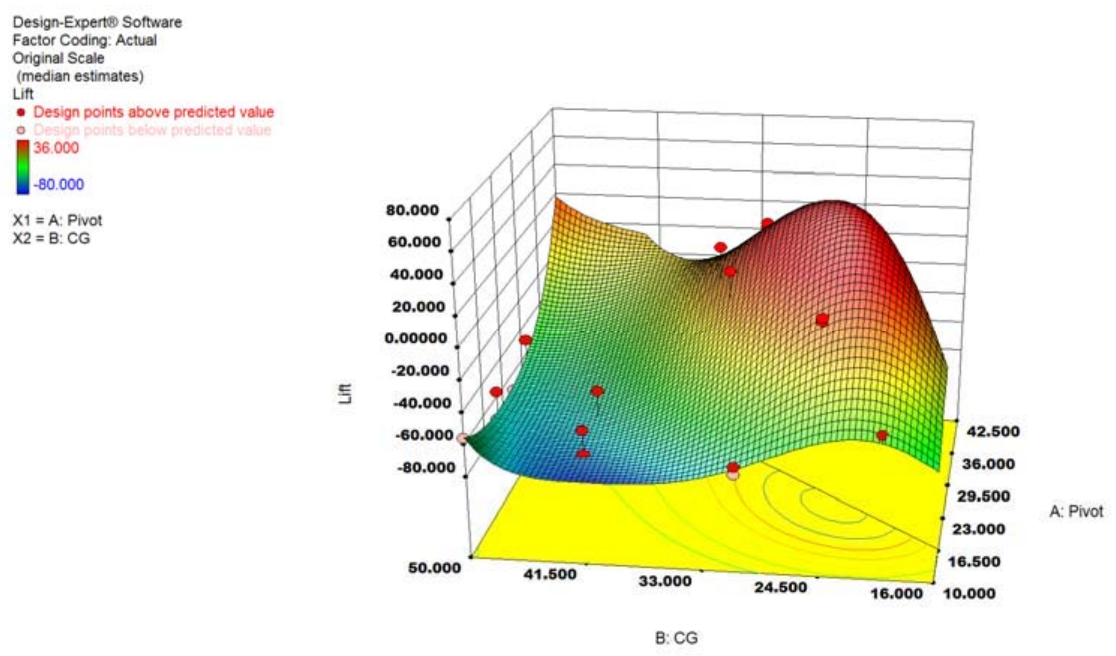

Figure 2. Lift optimization as a function of c.g. location and pivot point (blue area is better).

While the information in Figure 2 is somewhat useful, a reduced-order response surface allows for a better evaluation of some of the more salient characteristics observed. Specifically, as can be seen in Figure 3, countor plots of the reduced-order response surfaces to the primary variables indicate the region of the highest net lift is in the upper left corner, corresponding to the most forward pivot location and the most aft c.g. location. However, as

4

American Institute of Aeronautics and Astronautics 
can be seen in Figure 4, there is a different "sweet spot" associated with the roll authority available (the magnitude of the difference between the lift with the control surface full down vs. full up).

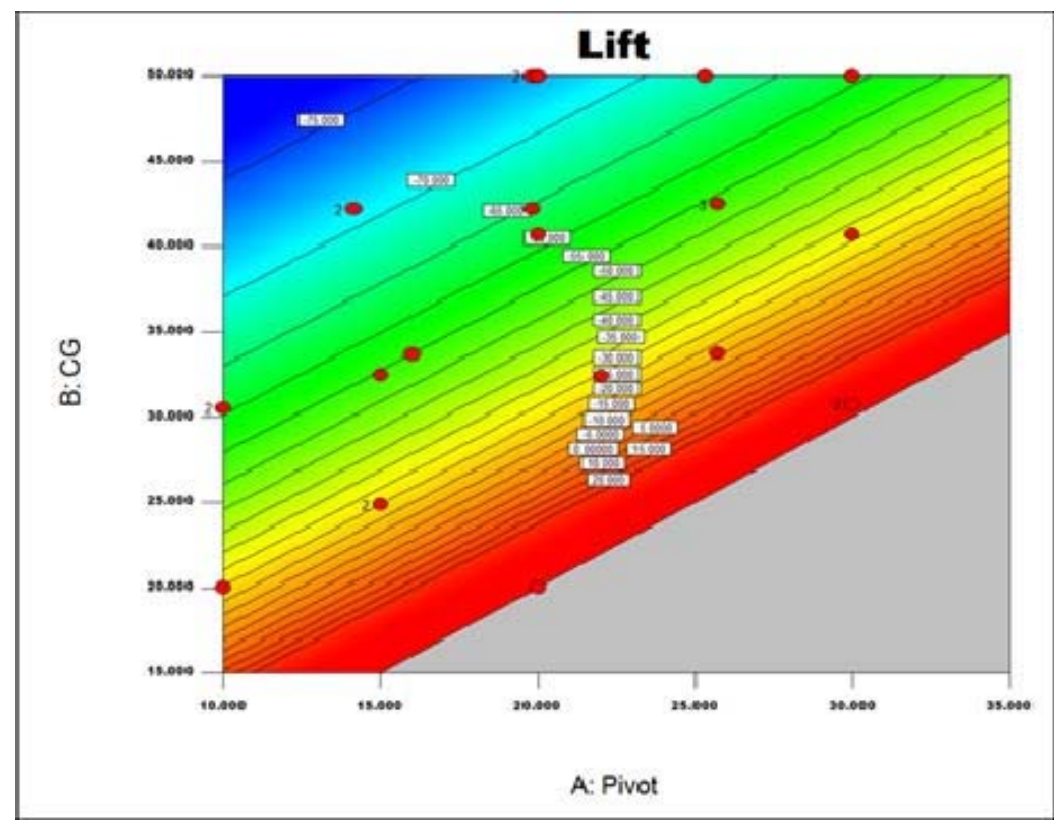

Figure 3. Lift output as a function of pivot and c.g. location (blue is better).

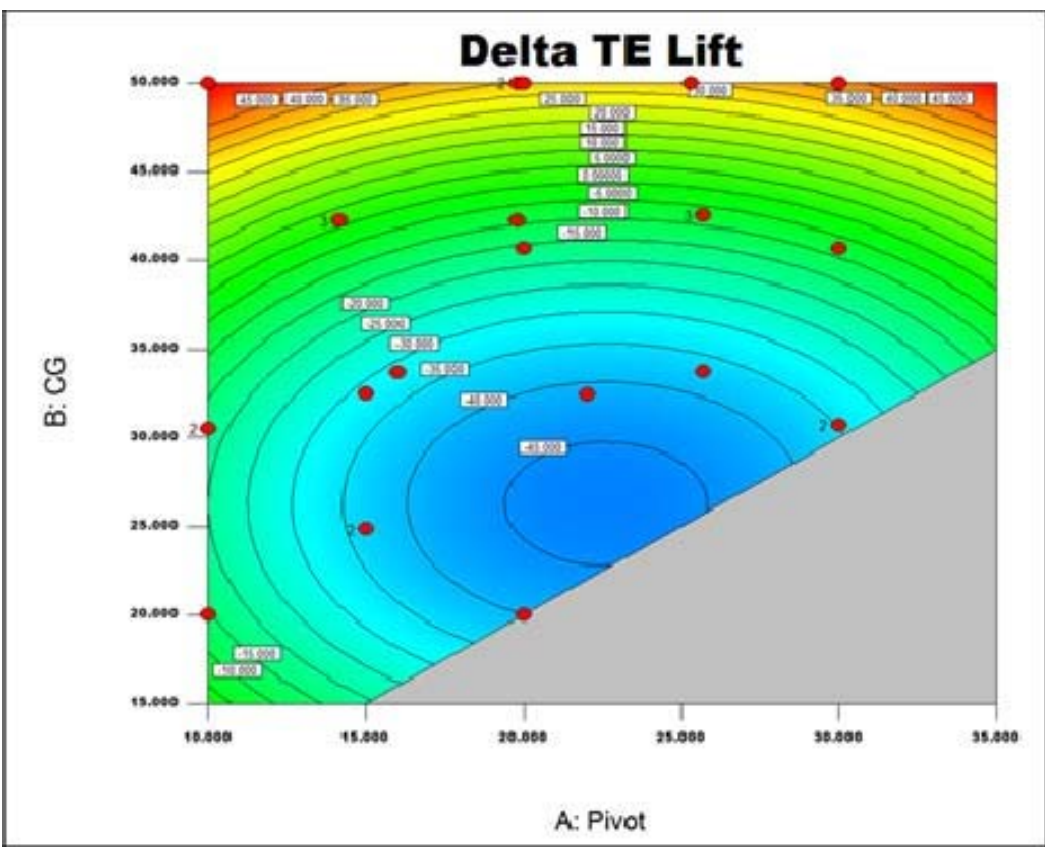

Figure 4. Effective roll authority as a function of pivot and c.g. location.

5

American Institute of Aeronautics and Astronautics 
It is therefore unclear where the "balance" should be between best lifting performance (most forward pivot, most aft c.g, i.e. farthest apart) and effective roll authority (which is at moderate pivot and c.g. locations close to one another). Assessment of this tradeoff will likely not be resolved until flight testing is conducted to discern where the level of roll authority is acceptable which minimizes the lift penalties. Further, flight testing in actual gusty conditions will need to be conducted to assess the degree to which the basic performance and roll authority "optimization” impact the ability to absorb large and small amplitude gusts in the target vehicle.

\section{Conclusion}

The results of the experiments conducted to date indicates that there is likely a tradeoff between better wing performance and roll authority for the free-to-rotate wing segments. Generically broad use of these data is not advised since the experiments were only conducted at one airspeed with one type of wing/airfoil. Flight tests will be needed to assess a "least non-optimum" set of pivot location and panel c.g characteristics which will provide adequate performance, roll control, and gust alleviation.

\section{Acknowledgments}

The authors would like to thank the NASA Aeronautics Seedling fund for providing the funds required to support this effort. The Aeronautics Seedling fund web site can be found at: http://nari.arc.nasa.gov/Seedling .

\section{References}

${ }^{1}$ Zuck, D.R., Patent \# 2347230, “Airplane with nonstalling and glide angle control characteristics”, 1944.

${ }^{2}$ Strand, T., and Levinsky, E., "Wind Tunnel Tests of a Free-Wing Tilt-Propeller V/STOL Airplane Model”, AFFDL-TR-6980, 1969.

3 Porter, R., and Brown, J., "Evaluation of the Gust-Alleviation Characteristics and Handling of a Free-Wing Aircraft", NASA CR-1523, 1970.

4 Porter, R. Luce, R., Brown, J., "Investigation of the Applicability of the Free-Wing Principle to Light, General Aviation Aircraft”, NASA CR-2046, 1972.

5 Porter, R., Hall, D., Brown, J., Gregorek, G., “Analytical Study of a Free-Wing/Free-Trimmer Concept”, NASA CR-2946, 1978.

6 Gee, S., Brown, S., "Flight Tests of a Radio-Controlled Airplane Model with a Free-Wing, Free-Canard Configuration”, NASA TM-72853, 1978.

7 Sandlin, D., “Wind Tunnel Tests of a Free-Wing/Free-Trimmer Model”, NASA CR-170394, 1982. 




